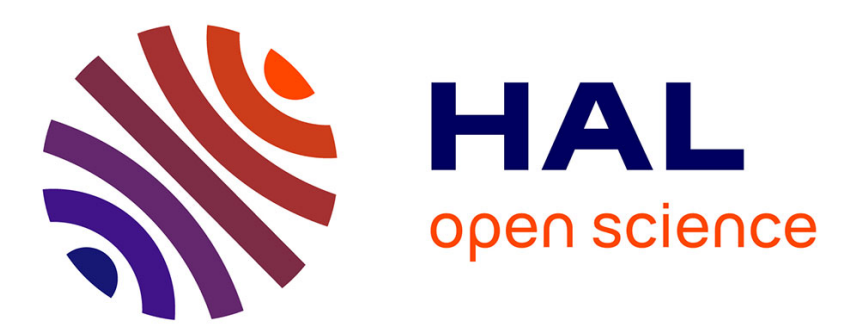

\title{
Advanced shape context for plant species identification using leaf image retrieval
}

Sofiène Mouine, Itheri Yahiaoui, Anne Verroust-Blondet

\section{To cite this version:}

Sofiène Mouine, Itheri Yahiaoui, Anne Verroust-Blondet. Advanced shape context for plant species identification using leaf image retrieval. ICMR '12 - 2nd ACM International Conference on Multimedia Retrieval, Jun 2012, Hong Kong, China. 10.1145/2324796.2324853 . hal-00726785

\section{HAL Id: hal-00726785 \\ https://hal.inria.fr/hal-00726785}

Submitted on 5 Feb 2013

HAL is a multi-disciplinary open access archive for the deposit and dissemination of scientific research documents, whether they are published or not. The documents may come from teaching and research institutions in France or abroad, or from public or private research centers.
L'archive ouverte pluridisciplinaire HAL, est destinée au dépôt et à la diffusion de documents scientifiques de niveau recherche, publiés ou non, émanant des établissements d'enseignement et de recherche français ou étrangers, des laboratoires publics ou privés. 


\section{Advanced shape context for plant species identification using leaf image retrieval}

\author{
Sofiene Mouine \\ INRIA Paris-Rocquencourt \\ 78153 Le Chesnay, France \\ sofiene.mouine@inria.fr
}

\author{
Itheri Yahiaoui \\ INRIA Paris-Rocquencourt \\ 78153 Le Chesnay, France \\ itheri.yahiaoui@inria.fr
}

\author{
Anne Verroust-Blondet \\ INRIA Paris-Rocquencourt \\ 78153 Le Chesnay, France \\ anne.verroust@inria.fr
}

\begin{abstract}
This paper presents a novel method for leaf species identification combining local and shape-based features. Our approach extends the shape context model in two ways. First of all, two different sets of points are distinguished when computing the shape contexts: the voting set, i.e. the points used to describe the coarse arrangement of the shape and the computing set containing the points where the shape contexts are computed. This representation is enriched by introducing local features computed in the neighborhood of the computing points. Experiments show the effectivness of our approach.
\end{abstract}

\section{Keywords}

Image retrieval, plant species identification, context-based recognition, shape context

\section{INTRODUCTION}

Identifying a plant can be a tricky task even for experienced botanists, considering the huge number of species existing in the world. This task is of great importance for a number of professionals such as land managers, foresters, agronomists, etc., and can be also useful for amateur gardeners. Plant identification is generally based on the observation of the morphological characteristics of the plant (such as general character, structures of stems, roots and leaves, embryology and flowers) followed by the consultation of a guide or a known database. An important amount of information about the taxonomic identity of a plant is contained in its leaves. Moreover, leaves are present on the plants for several months in a year, whereas flowers and fruits may remain only several weeks. This is why most plant identification tools based on Content-Based Image Retrieval techniques work on leaf image databases $[17,29,20,24,31,10$, $4,7,23,25,6,3,8,9,13]$. A leaf can be characterized by its color, its texture, and its shape. The color of a leaf may vary with the seasons and climatic conditions. In addition, as most plants have similar colors, this feature is not

Permission to make digital or hard copies of all or part of this work for personal or classroom use is granted without fee provided that copies are not made or distributed for profit or commercial advantage and that copies bear this notice and the full citation on the first page. To copy otherwise, to republish, to post on servers or to redistribute to lists, requires prior specific permission and/or a fee.

ICMR '12, June 5-8, Hong Kong, China

Copyright (C)2012 ACM 978-1-4503-1329-2/12/06 ...\$10.00. discriminant enough for the species recognition. Thus, generally, only shape and texture information are taken into account in similarity based leaf image retrieval schemes.

Several techniques have been introduced to solve the problem of automatic leaf identification.

Existing methods generally use a shape-based approach. This is not the case for [13] where shape and texture descriptors on oriented patches centered around Harris points are computed and a large scale matching method [16] performs the leaf identification. No prior segmentation is made and Harris points are not necessarily located on the leaf margin. This approach is generic and works well on scans of leaves [14] but may be fastidious for images with a cluttered background. Shape-based approaches mainly work on the overall shape or on the contour of the leaves.

A first group of methods extracts morphological plant characters commonly used in botany. Du et al. [10] compute eight features, Aspect Ratio, Rectangularity, Convex Area Ratio, Convex Perimeter Ratio, Sphericity, Circularity, Eccentricity and Form Factor, from the boundary of the leaves. Morphological features are also retained and used in the identification process in the parameterized segmentation representation of leaves proposed by Cerruti et al. [9]. Eccentricity is used in the two-stage approach of Wang et al. [29] and of Caballero and Aranda [7] to reduce the search space. Other shape feature extraction techniques [19] have been adapted or introduced to solve the plant retrieval problem. Neto et al. [24] used elliptic Fourier functions on the leaf shape. Statistical methods based on the method of Complex Networks $[3,8]$ on the contour of the leaf or extracting fractal dimension [6] on the contour and the venation of the leaf have been proposed. Yanikoglu et al. [32] obtain good results on leaf scans with a combination of texture, shape and color descriptors among which some are based on algorithms of mathematical morphology. Different types of $2 \mathrm{D}$ histograms using geometric features, such as curvature, lengths, relative orientation, distances, etc., computed on the boundary points of the leaf have been presented in [31, $18,7]$. In [31], a 2D directional fragment histogram computes directions and relative lengths on a succession of elementary fragments on the contour. Another 2D histogram derived from the shape context [5] computing inner distances and angles between sample points of the leaf margin is proposed in $[18,4]$. Curvature of the leaf contour is used in the Curvature Scale Space representation [21, 1] and in Caballero and Aranda 's approach [7]. Note that the shape features computed from the leaf margin can be enriched with venation features [25, 23]. Most of these shape-based ap- 
proaches are adapted to the leaf identification problem, but their effectiveness may depend on the quality of the contour obtained by the segmentation process.

In this paper, we combine local and shape-based features to obtain an efficient and effective leaf identification method. For this purpose, we select appropriate salient points of the leaf and model local information and spatial relations by a shape context based approach [5].

This paper is structured as follows. A family of shape context based approaches is introduced in section 2. They work on different sets of selected points of the leaf. Local features are then introduced in our model to enrich the image description. Experimental results are presented in section 3 . The last section concludes the paper and presents our future work.

\section{ADVANCED SHAPE CONTEXT}

Shape context technique [5] has proven its efficiency for shape retrieval, even for leaf images, with the inner shape context $[18,4]$. To describe properly the boundary of a shape and obtain good retrieval results, a dense sampling of the contour points is necessary. Then a large number of histograms are computed and compared, making the overall technique expensive. To solve this problem, Xie et al. [30] have introduced the skeletal shape context, which uses a medial axis transform to produce an optimal sampling of the shape contour with a smaller number of points.

Shape context retains only global shape information of an image region. To enrich this description Amores et al. [2] have extended the shape context approach for object classrecognition, introducing color and edge information in the histogram. They first perform region segmentation and use the region boundaries as contours of the image. Their "generalized correlograms", encoding local and spatial information, are computed on a sampled set of contour points. In our case, we have a unique class-object, the Leaf class, and our goal is to identify the species of a leaf, which can be characterized by its venation, its margin and its texture. We also retain local information in the image description (see Section 2.2) but we use here another strategy to encode the spatial information in the images.

In our shape context based approach, we want to reduce the computational cost while preserving or increasing the shape matching precision. We think that introducing two different sets of points that play different roles in the shape context scheme and choosing them appropriately will help us to achieve this goal. Thus we distinguish the voting points, which is the set of points used to build the shape context histograms from the computing points on where the shape contexts are computed.

In fact, the computing points correspond to characteristic or salient points of the object and, for efficiency purposes, the cardinality of this set has to be low. The voting points are other points belonging to the object and must add information on the shape when involved in the computation of shape context on the computing points. Here, as these points are used only once for each histogram, we can use a representative number of voting points. For example, for a polygonal shape, the computing points can be the extrema of boundary curvature points and the voting points the boundary points. Let us now present our approach in more details.

\subsection{Advanced shape context}

Given a set of $n$ points $\mathcal{V}$ and a point $p$ of $\mathbb{R}^{2}$, the advanced shape context of $\mathcal{V}$ on $p$ is a discrete representation of the set of $n$ vectors defined by the pairs of points $(p, q)$ with $q \in \mathcal{V}$. It is represented by a coarse histogram $a S C(p, \mathcal{V})$ where each pair of points $(p, q)$, represented by a radius $r$ and an angle $\theta$, contributes to the bin $k$ using the log-polar quantization introduced in [5] and used more recently in [2, $18,28]$.

$$
a S C(p, \mathcal{V})_{k}=\#\left\{q \in \mathcal{V}: q-p \in \operatorname{bin}_{p}(k)\right\}
$$

In our implementation, the radius is quantized into 5 bins and the angle into 12 bins.

In the rest of this paper, the set $\mathcal{V}$ is denoted the voting set of points and the set $\mathcal{C}$ of points $p$ of $\mathbb{R}^{2}$, where the advanced shape context $a S C(p, \mathcal{S})$ is computed, is called the computing set. The sets $\mathcal{C}$ and $\mathcal{V}$ are not necessarily distinct.

Let us now return to our application.

We suppose in this paper that a leaf image consists in a leaf picture with a nearly white uniform background. A leaf is not only characterized by its margin: its venation network may be significant. Some plant species have a high intra-variability of the leaf shape, and leaves from different species may have globally similar shapes as shown in figure 1. Thus, in this case the venation network information may
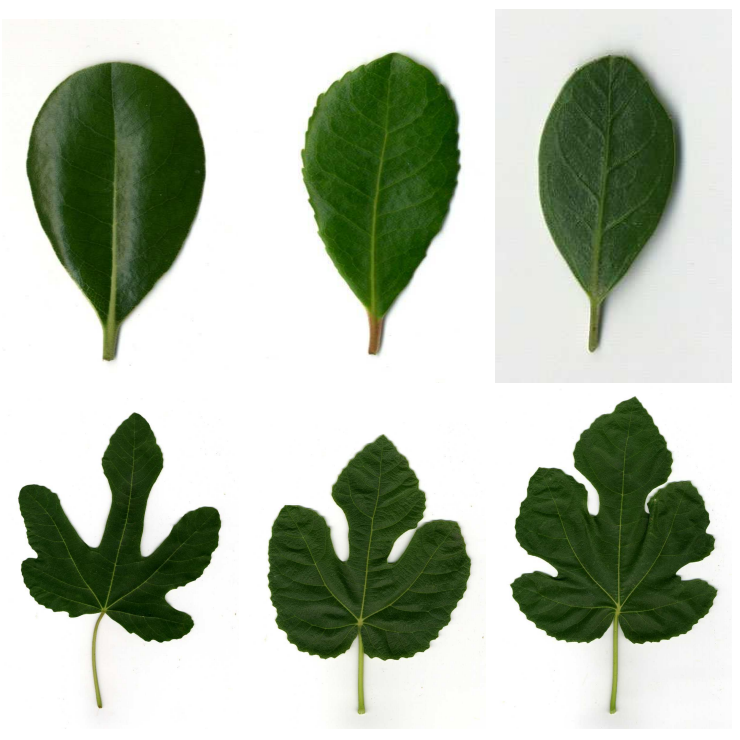

Figure 1: Top row: Overall shape similarity between different species (Pittosporum tobira, Arbutus unedo, Rhamnus alaternus). Bottom row: Intra-variability of the species Ficus carica

be useful for an identification task.

In fact, salient points on the margin are extrema of curvature when leaves are lobed or toothed and are also junction points when the venation network can be extracted from the image.

In our case:

- The leaf margin is a closed contour calculated by using the Otsu thresholding method since the image background is homogeneous.

- The salient points are computed with the generic Harris detector, which is known to detect edge corners efficiently. 
In order to test our approach on images of leaves, three scenarios are proposed by varying the computing set $\mathcal{C}$ and the voting set $\mathcal{V}$ of points in the image.

SCO Spatial relations between margin points. Here the computing set $\mathcal{C}$ and the voting set $\mathcal{V}$ are identical. They involve the margin points, i.e. $n$ points extracted from the margin by a uniform quantization.

$$
\mathcal{C}=\mathcal{V}=\{\text { margin points }\}
$$

This scenario corresponds to the shape context proposed by Belongie et al. [5]. Note that the venation network is not introduced here.

\section{SC1 Spatial relations between salient points.}

As in the previous case, $\mathcal{C}$ and $\mathcal{V}$ represent the same set. They both contain $n$ salient points computed with the Harris corner detector.

$$
\mathcal{C}=\mathcal{V}=\{\text { salient points }\}
$$

This scenario is similar to the logo retrieval approach [27]. The goal here is to determine whether the spatial relationships between salient points on the leaf area can characterize leaves of a given species and to evaluate the influence of the number of considered salient points on the plant identification performance.

SC2 Spatial relations between salient and margin points

Here we want to measure the spatial relationships between the salient points described in the context defined by the leaf margin. The voting set of points $\mathcal{V}$ is composed of all the margin points. The Harris points form the computing set $\mathcal{C}$.

$\mathcal{C} \neq \mathcal{V}, \mathcal{C}=\{$ salient points $\}$ and $\mathcal{V}=\{$ margin points $\}$

As mentioned above, the salient points may lay inside the leaf or may belong to the leaf margin. Our aim is to study the correlation between the venation network and the margin of the leaves belonging to the same species.

\subsection{Local properties}

The advanced shape context captures a spatial configuration of points without taking into account local properties of the image around the set $\mathcal{C}$ of computing points. Thus, to enrich the description, a set of local features computed on the neighborhood of each point of $\mathcal{C}$ is introduced. As colors are not a discriminant feature for leaves, we focus on texture and shape. To describe the texture and the shape, three local features are extracted from the grey-level of an image patch located around each computing point:

- A 16 dimensional Hough histogram, hough_4_4, based on the Hough transform, is used to represent simple shapes in an image [11]: for each pixel, the gradient orientation and the projection amplitude of the pixel vector onto the tangent vector to the local edge are used to build a $2 \mathrm{D}$ histogram.

- A Fourier histogram, fourier_8_32 [11], based on the Fourier transform, which contains information about texture and scale: two histograms are computed in the complex plane from the Fourier transform. They represent two types of distributions of the energy: the first one is computed with a circular partition, the second uses a wedge partition. Both have an equal importance in the final signature, which gives a description of the energy in the image at several scales as well as a description of the local small scale behavior in a number of predefined directions.

- A 8 dimensional classical Edge Orientation Histogram, which is known to be suitable for non-uniform textures.

These three features have given promising results when associated with Harris points on scans of leaves in [14].

In the following, the combination of the Hough, the Fourier and the edge orientation histogram is denoted by std.

\subsection{Matching Method}

The matching process is the same for all the scenarios. Let $I$ be an image and let $n$ be the number of points of $I$ in the computing set $\mathcal{C}_{I}$. I is represented by $n$ feature vectors $F_{1}, F_{2}, \ldots, F_{n}$ where $F_{i}$ is the feature vector associated to the $i^{t h}$ point of $\mathcal{C} . F_{i}$ is of dimension $k$ with:

$$
k=n \text { bins }_{r} \times n \text { bins }_{\theta} \text { or } k=\text { nbins }_{r} \times n b i n s_{\theta} \times n_{s t d}
$$

where bins $_{r}$ and $n$ bins $s_{\theta}$ are respectively the number of bins for quantified log-polar distances and the number of bins for quantified angles and $n_{s t d}$ is the size of the std feature vector presented in Section 2.2.

The features matching, is done by an approximate similarity search technique based on a Locality Sensitive Hashing (LSH) method [26]. We use here the Multi Probe Locality Sensitive Hashing technique proposed by Joly and Buisson [15] and the distance $L_{2}$ to compute the similarity between two feature vectors. The principle of this algorithm is to project all the features in an $\mathrm{L}$ dimensional space and to use hash functions to reduce the search and the cost time. At query time, the features $F_{1}, F_{2}, \ldots, F_{n}$ of the query image are mapped onto the hash tables and the knn nearest neighbors of each feature $F_{i}$ are searched in the buckets associated to $F_{i}$. These $n$ lists of candidate feature matches are used as input for a voting system to rank images according to the number of matched features.

One can notice here that if scenario SC0 has the same histograms as the shape context methods [5, 22], the overall approach is not identical, as our matching method is different. It is the same for the SC1 scenario and the the logo retrieval approach of [27] as, in their case, the LSH approximate search is performed on shapeme histograms.

\section{EXPERIMENTAL RESULTS}

All the approaches presented above have been tested on a set of leaf images acquired using a flat-bed scanner. This is the training subset of Pl@ntLeaves dataset that was used for the plant identification task organized within ImageCLEF 2011 [12]. This set contains 2349 images of scans of leaves. We also compare our results with the scores of identification of ImageCLEF2011 obtained on two categories of images: scans and scan-like leaf images. Figure 2 gives a view of the different species present in the database.

To evaluate the three scenarios SC0, SC1 and SC2, we use the precision $P$ and recall $R$ measures defined respectively by

$$
\begin{gathered}
P=\frac{\# \text { relevant images }}{\# \text { retrieved images }} \text { and } \\
R=\frac{\# \text { retrieved relevant images }}{\# \text { relevant images }}
\end{gathered}
$$




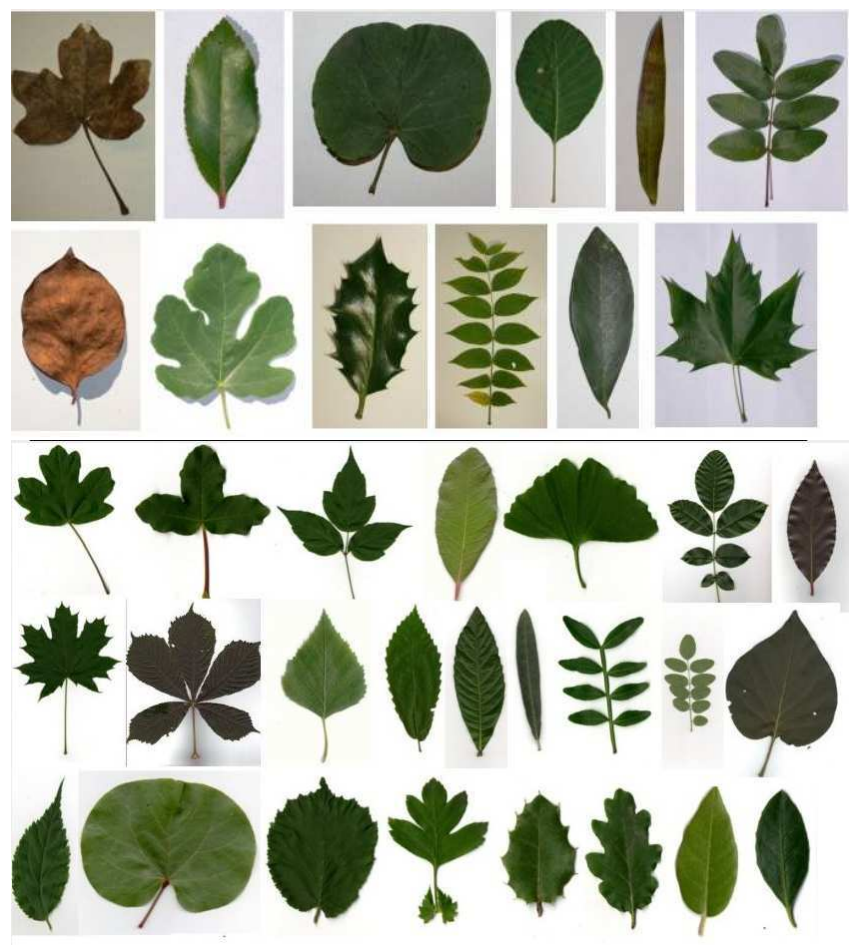

Figure 2: A selection of scan-like leaves (first two rows) and scan leaves of the Pl@ntNet test dataset. (Only one leaf per species is kept)

and the Mean Average Precision (MAP). It is measured on a set of queries $Q$ and is defined as follows:

$$
M A P=\frac{\sum_{q=1}^{Q} A P(q)}{Q}
$$

where the average precision score $A P$ is computed for each query $q$ :

$$
A P=\frac{\sum_{k=1}^{n}(P(k) \times f(k))}{\# \text { retrieved relevant images }}
$$

$f(k)$ takes the value 1 if the returned image at rank $k$ is relevant, zero otherwise. The MAP value is correlated with the precision value $P$. However, it gives a more general result taking into account all the images of the database.

\subsection{Results on the training scan dataset}

Scenarios SC0, SC1, SC2 have been tested with computing sets of points of cardinality 50 and 400. Precision Recall curves are shown in Figure 3. The std curve corresponds to the retrieval using only Harris points associated with local features around them. Enriched SC2 is presented by the curve SC2+std. Examining these precision-recall curves and the MAP values of Table 3.1, we can notice that:

- All the scenarios give better results with 400 points compared to 50 points.

- The information provided by the Scenario SC1 is less meaningful compared with other scenarios. This is due to the fact that, when only 50 Harris points are computed, they provide
Table 1: MAP values for the different scenarios

\begin{tabular}{|c|c|c|c|c|c|}
\hline Approach & SC0 & SC1 & SC2 & std & SC2+std \\
\hline MAP (50points) & 0.36 & 0.14 & 0.40 & 0.45 & 0,50 \\
\hline MAP (400points) & 0.40 & 0.25 & 0.42 & 0.57 & 0,68 \\
\hline
\end{tabular}

a very coarse representation of the margin and the venation network.

- Scenario SC2 gives the best $\mathrm{R} / \mathrm{P}$ curve and the MAP value compared to SC1 and SC0. However, the std method that computes local features around Harris points, is more effective than SC2. Moreover, the results are improved when local features are associated to Harris points in scenario SC2, which corresponds to the enriched SC2 (SC2+std).

- SC2 obtains good results with a small number of computing points. When $\mathrm{SC} 2$ is used with only 50 points, it gives similar performances to SC0 with 400 computing points.

- The enriched SC2 (SC2+std) outperforms all the other scenarios. It has the best Recall/Precision curves and the best MAP values. When 400 computing points are used, the MAP value of $\mathrm{SC} 2$ is 0.68 . This proves that the joint use of spatial information and local information increases the identification rate on the training Scan dataset.

Let us compare the retrieval results obtained using scenarios SC0 and SC2 on a Rhamnus alaternus leaf in Figure 4. With SC0, only three images are relevant among the first 15 returned images which gives a precision $P=20 \%$ for $k n n=15$. In fact, the overall shape of all the returned leaves is very similar. Consequently, additional information about the leaf taxonomy is needed to be robust to shape similarity between the species.

This was our first motivation to build SC2 by separating two sets of points. Figure 4(b) shows the retrieval response using SC2 without including local features. The precision for the first 15 returned images is $P=80 \%$ which is much higher than the results of SC0. This can be explained by the fact that SC2 includes, with the use of salient points, informative characters within the leaf area such as venation, texture, etc. Note that the information on the contour is not lost in this schema since the voting set contains points of the leaf margin. Moreover, salient points computed with a Harris detector may be located on the boundary, in particular, for the toothed leaves contour.

In Figure 5, we show two retrieval results with the same query image of Figure 4 . In the case of $\mathrm{SCO}$, an important
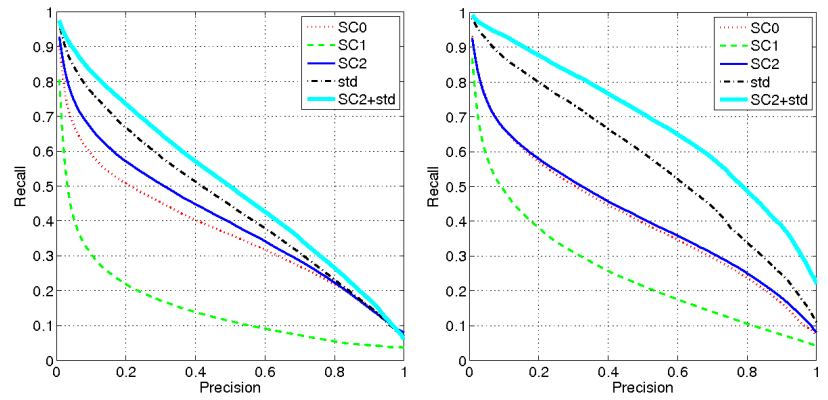

Figure 3: Recall/Precision curves on the training scan dataset using 50 points (Left) and 400 points (right) 


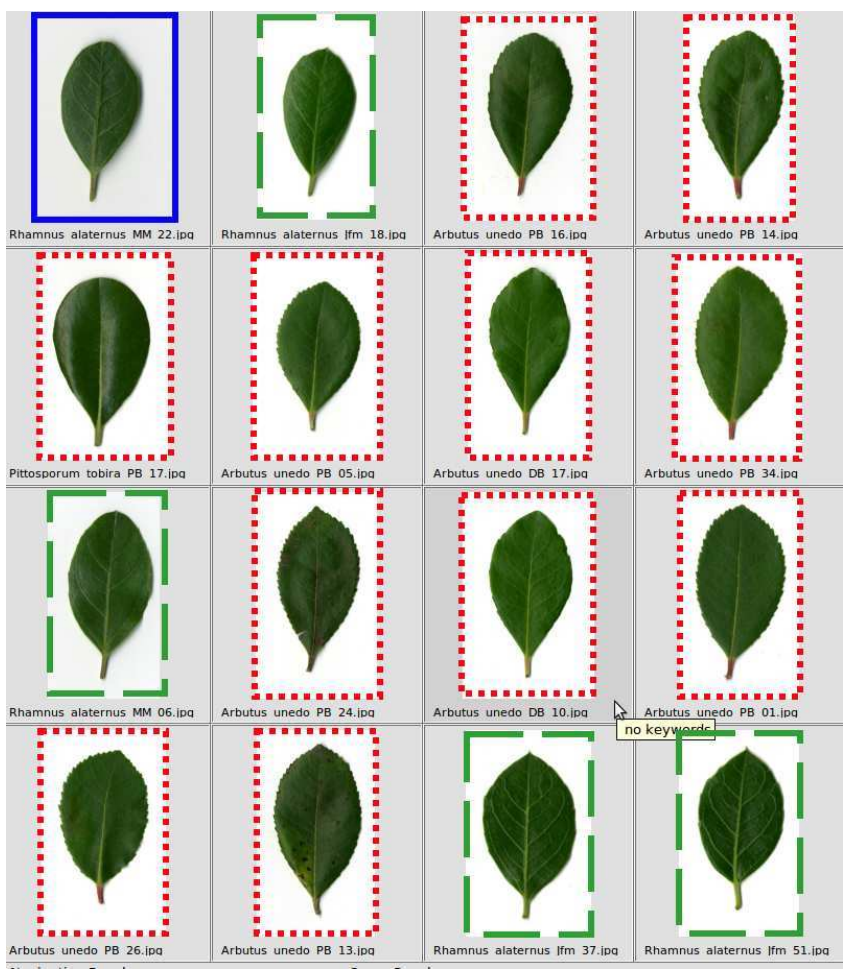

(a)

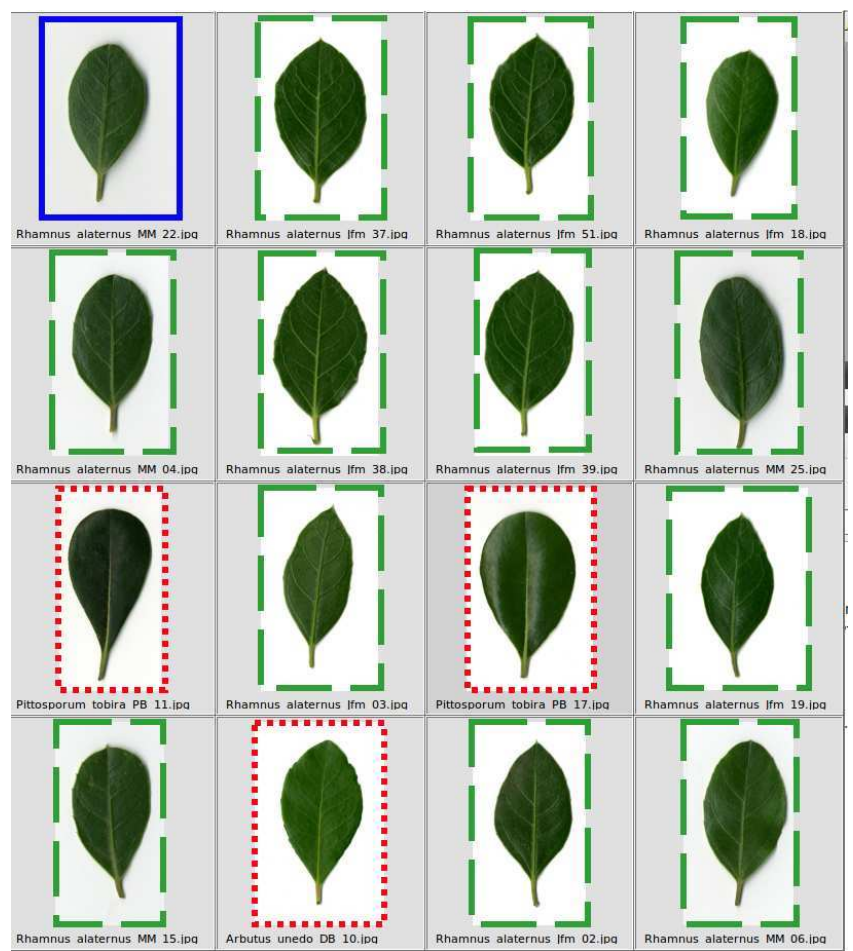

(b)

Figure 4: Two retrieval responses with the same query image using SC0 for (a) and SC2 for (b). Query image $Q$ is framed by a solid line, relevant retrieved images i.e leaves from the same species of $\mathrm{Q}$ are framed by a dashed line and false positive images are framed by a dotted line

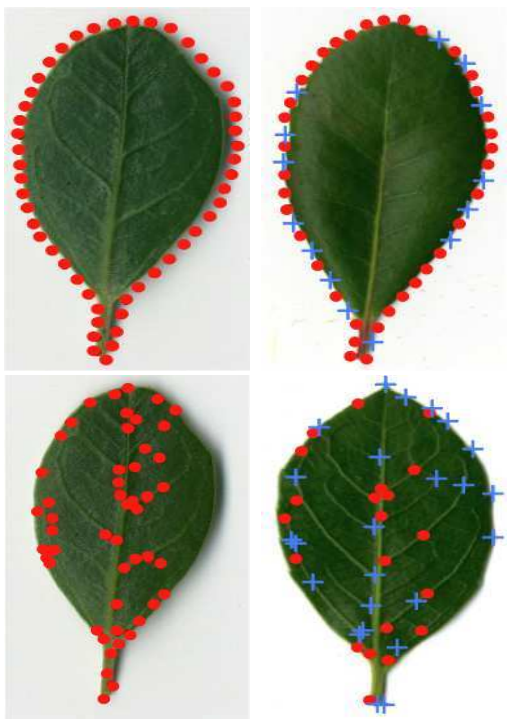

Figure 5: Top row: the query image used in Figure 4 and a response example using SC0, red points on the right image are the points that matched with contour points of the query image. Bottom row: the query image used in Figure 4 and a response example using SC2, red points on the right image are the points that matched with Harris points of the query image

number of points of the returned image matched with the contour points of the query image. However, the returned image belongs to another species. In fact, the entire configuration of the shape is very similar but the venation network is different. Thus, the spatial representation provided by SC0 may not be appropriate in this particular case. On the other hand, matched points computed with $\mathrm{SC} 2$ are either on the nervation, on the contour or inside the leaf. The main advantage of SC2 is that the contour is not considered as an exclusive source of information. Here, the retrieved image and the query image belong to the same species.

\subsection{Comparison with ImageCLEF2011 results}

Let us first introduce the context of the organized identification task ImageCLEF 2011[12]. The goal of the task was to find the correct tree species of each test image. The identification score is quite different from the classic measures presented above such as the MAP value and recall-precision curves. Two assumptions guided the identification score $S$ definition:

- The leaves from the same tree may be more similar than leaves from different trees.

- Photos taken by the same person will have nearly the same acquisition protocol.

So, $S$ is defined as follows in ImageCLEF 2011:

$$
S=\frac{1}{U} \sum_{u=1}^{U} \frac{1}{P_{u}} \sum_{p=1}^{P_{u}} \frac{1}{N_{u, p}} \sum_{p=1}^{N_{u, p}} s_{u, p, n}
$$

$U$ : number of users (who have at least one image in the test data).

$P_{u}$ : number of individual plants observed by the $u^{t h}$ user. 
Table 2: Species appearing either on the test scan or on the scan-like dataset versus the train scan dataset on Pl@ntLeaves database of ImageCLEF 2011

\begin{tabular}{|c|c|c|c|}
\hline species & TeSL & $\mathrm{TeS}$ & $\operatorname{TrS}$ \\
\hline Acer campestre & 9 & 22 & 24 \\
\hline Acer monspessulanum & & 22 & 45 \\
\hline Acer negundo & & 21 & 17 \\
\hline Acer platanoides & & 10 & 12 \\
\hline Aesculus hippocastanum & & 4 & 25 \\
\hline Albizia julibrissin & & & 45 \\
\hline Alnus glutinosa & & & 8 \\
\hline Arbutus unedo & 1 & 41 & 34 \\
\hline Betula pendula & & 3 & 76 \\
\hline Carpinus Betulus & & & 33 \\
\hline Castanea sativa & & 45 & 24 \\
\hline Celtis australis & & 24 & 39 \\
\hline Cercis siliquastrum & 11 & 20 & 49 \\
\hline Corylus avellana & & 20 & 55 \\
\hline Cotinus coggygria & 29 & & 64 \\
\hline Crataegus azarolus & & & 38 \\
\hline Crataegus monogyna & & 54 & 23 \\
\hline Diospyros kaki & 7 & & 43 \\
\hline Eriobotrya japonica & & 21 & 14 \\
\hline Fagus sylvatica & & & 28 \\
\hline Ficus carica & 19 & & 43 \\
\hline Fraxinus angustifolia & & & 74 \\
\hline Fraxinus ornus & & & 33 \\
\hline Ginkgo biloba & & 15 & 34 \\
\hline Gleditsia triacanthos & & & 32 \\
\hline Ilex aquifolium & 26 & & 40 \\
\hline Juglans nigra & 22 & & 16 \\
\hline Juglans regia & & 4 & 30 \\
\hline Laburnum anagyroides & & & 30 \\
\hline Laurus nobilis & 16 & 6 & 36 \\
\hline Ligustrum vulgare & & & 26 \\
\hline Magnolia grandiflora & & & 23 \\
\hline Malus sylvestris & & & 17 \\
\hline Nerium oleander & 8 & & 88 \\
\hline Olea europaea & & 25 & 125 \\
\hline Paliurus spina-christi & & 7 & 65 \\
\hline Phillyrea angustifolia & & & 15 \\
\hline Pistacia lentiscus & & 36 & 41 \\
\hline Pistacia terebinthus & & & 47 \\
\hline Pittosporum tobira & & & 67 \\
\hline Platanus x hispanica & 2 & & 49 \\
\hline Prunus mahaleb & & & 49 \\
\hline Prunus serotina & & & 24 \\
\hline Prunus spinosa & & & 40 \\
\hline Punica granatum & & & 38 \\
\hline Quercus coccifera & & 33 & 16 \\
\hline Quercus ilex & 29 & 18 & 123 \\
\hline Quercus petraea & & & 14 \\
\hline Quercus pubescens & & 18 & 24 \\
\hline Rhamnus alaternus & & 52 & 54 \\
\hline Rhamnus cathartica & & & 27 \\
\hline Robinia pseudoacacia & & 26 & 32 \\
\hline Salix caprea & & & 26 \\
\hline Sambucus nigra & & & 9 \\
\hline Sophora japonica & & & 32 \\
\hline Sorbus domestica & & & 21 \\
\hline Syringa vulgaris & & 20 & 51 \\
\hline Tilia cordata & & & 23 \\
\hline Viburnum lantana & & & 17 \\
\hline Viburnum tinus & & 94 & 47 \\
\hline Vitex agnus-castus & & 2 & 55 \\
\hline
\end{tabular}

\begin{tabular}{|r|l|l|l|}
\hline & $\begin{array}{l}\text { Number of } \\
\text { images }\end{array}$ & $\begin{array}{l}\text { Number of } \\
\text { individual plants }\end{array}$ & $\begin{array}{l}\text { Number of } \\
\text { contributors }\end{array}$ \\
\hline Scan train & 2349 & 151 & 17 \\
test & 721 & 55 & 13 \\
\hline test & 180 & 51 & 2 \\
Scan-like train & 717 & 1 \\
\hline
\end{tabular}

Table 3: Statistics of the composition of the training and test data of the Pl@ntLeaves dataset

$N_{u, p}$ : number of pictures taken from the $p^{\text {th }}$ plant observed by the $u^{t h}$ user.

$s_{u, p, n}$ : classification score $(1$ or 0$)$ for the $n^{t h}$ picture taken from the $p^{\text {th }}$ plant observed by the $u^{\text {th }}$ user.

We focus on scans and scan-like images. Table 2 and Table 3 describe the number of images and their distribution between the different datasets.

The evaluation metric $S$ is used to compare our approach with the others. Only the top 10 scores of ImageCLEF2011 are presented in Table 4. More details about the methods and the complete list of scores can be found in [12]. We present here the score of SC0 and SC2 using 50 computing points.

Both SC0 and SC2 outperform all the other approaches on the scan-like dataset. We obtain the best identification score using SC0 $(S=0.706)$. SC2 is also better than the top ten scores of ImageClef with a score $S=0.677$. In fact, Harris points can be located on the background for the scan-like images. That is why the spatial description provided by SC2 is less accurate than SC0.

We also obtain good results on the scan images. SC2 performs better than $\mathrm{SC} 0$ on the scans and they are respectively positioned in the third and the fourth place with respect to ImageCLEF 2011 runs.

In scans and scan-like dataset, $\mathrm{SC} 2+$ std which had the best result on the training scan dataset loses its first position. It can be explained by the fact that the combination of local and spatial relationships between Harris points is more efficient to retrieve almost identical images more than similar ones. It is worth noting that the ImageCLEF measure is normalized according to the individual plant and the user.

Table 4: Normalized classification scores of the scan and scan-like images on the Pl@ntLeaves dataset using the evaluation metric of [12]

\begin{tabular}{|c|c|c|c|}
\hline run_id & Participant & Scans & Scan-like \\
\hline IFSC_USP_run2 & IFSC & $\mathbf{0 . 5 6 2}$ & 0.402 \\
inria_imedia_plantnet_run1 & INRIA & $\mathbf{0 . 6 5 8}$ & 0.464 \\
IFSC_USP_run1 & IFSC & 0.411 & 0.430 \\
LIRIS_run3 & LIRIS & 0.546 & 0.513 \\
LIRIS_run1 & LIRIS & 0.539 & $\mathbf{0 . 5 4 3}$ \\
Sabanci-okan-run1 & SABANCI-OKAN & $\mathbf{0 . 6 8 2}$ & 0.476 \\
LIRIS_run2 & LIRIS & 0.530 & 0.508 \\
LIRIS_run4 & LIRIS & 0.537 & $\mathbf{0 . 5 3 8}$ \\
inria_imedia_plantnet_run2 & INRIA & 0.477 & $\mathbf{0 . 5 5 4}$ \\
IFSC_USP_run3 & IFSC & 0.356 & 0.187 \\
\hline SC0 & & 0.654 & 0.706 \\
\hline SC2 & & 0.676 & 0.677 \\
\hline SC2+std & & 0.65 & 0.59 \\
\hline
\end{tabular}




\section{CONCLUSION}

In this paper, we have presented a new approach extending the shape context method for plant species identification on leaf images. To compute histograms, two sets of points have been introduced: the computing set and the voting set. Several scenarios based on different computing and voting sets have been proposed and tested on scans and scan-like leaf images. Scenarios SC0 and SC1 work respectively only on leaf margin points and only on salient points. Scenario SC2 uses both contour points and salient points of the leaf in the image representation. We have shown the effectiveness of the enriched $\mathrm{SC} 2$ on scans of leaves.

Furthermore, our approach has been compared favorably to ImageCLEF2011 approaches.

In this framework, we used a generic corner detector (Harris) to compute salient points on the images. Work in progress extends this approach in two ways:

- We are studying specific detectors for leaf salient points based on botanical expertise

- We are investing new scenarios using venation network and margin points.

\section{Acknowledgements}

This research has been conducted with the support of the Agropolis Foundation through the Pl@ntNet project. I would like also to thank Vera Bakić for her help to accomplish this work.

\section{REFERENCES}

[1] S. Abbasi, F. Mokhtarian, and J. Kittler. Reliable classification of chrysanthemum leaves through curvature scale space. In Scale-Space Theory in Computer Vision, volume 1252, pages 284-295. LNCS, 1997.

[2] J. Amores, N. Sebe, and P. Radeva. Context-based object-class recognition and retrieval by generalized correlograms. IEEE Transactions on Pattern Analysis and Machine Intelligence, 29(10):1818 -1833, Oct. 2007.

[3] A. R. Backes, D. Casanova, and O. M. Bruno. A complex network-based approach for boundary shape analysis. Pattern Recognition, 42(1):54-67, 2009.

[4] P. Belhumeur, D. Chen, S. Feiner, D. Jacobs, W. Kress, H. Ling, I. Lopez, R. Ramamoorthi, S. Sheorey, S. White, and L. Zhang. Searching the world's herbaria: A system for visual identification of plant species. In European Conference on Computer Vision (ECCV), pages 116-129, 2008.

[5] S. Belongie, J. Malik, and J. Puzicha. Shape matching and object recognition using shape contexts. IEEE Transactions on Pattern Analysis and Machine Intelligence, 24(4):509 -522, apr 2002.

[6] O. M. Bruno, R. de Oliveira Plotze, M. Falvo, and M. de Castro. Fractal dimension applied to plant identification. Information Sciences, 178(12):2722 2733, 2008.

[7] C. Caballero and M. C. Aranda. Plant species identification using leaf image retrieval. In $A C M$ International Conference on Image and Video Retrieval (CIVR), pages 327-334, 2010.

[8] D. Casanova, J. B. Florindo, and O. M. Bruno. IFSC/USP at ImageCLEF 2011: Plant identication task. In CLEF (Notebook Papers/Labs/Workshop), 2011.

[9] G. Cerutti, L. Tougne, A. Vacavant, and D. Coquin. A parametric active polygon for leaf segmentation and shape estimation. In International Symposium on Visual Computing (ISVC), pages 202-213, 2011.

[10] J.-X. Du, X.-F. Wang, and G.-J. Zhang. Leaf shape based plant species recognition. Applied Mathematics and Computation, 185(2):883 - 893, 2007.

[11] M. Ferecatu. Image retrieval with active relevance feedback using both visual and keyword-based descriptors. PhD thesis, University of Versailles Saint-Quentin-en-Yvelines, 2005.

[12] H. Goëau, P. Bonnet, A. Joly, N. Boujemaa, D. Barthelemy, J.-F. Molino, P. Birnbaum, E. Mouysset, and M. Picard. The CLEF 2011 plant images classification task. In CLEF (Notebook Papers/Labs/Workshop), 2011.

[13] H. Goëau, A. Joly, S. Selmi, P. Bonnet, E. Mouysset, L. Joyeux, J.-F. Molino, P. Birnbaum, D. Bathelemy, and N. Boujemaa. Visual-based plant species identification from crowdsourced data. In 19th ACM international conference on Multimedia, pages 813-814, 2011.

[14] H. Goëau, A. Joly, I. Yahiaoui, P. Bonnet, and E. Mouysset. Participation of INRIA\& Pl@ntNet to ImageCLEF 2011 plant images classification task. In CLEF (Notebook Papers/Labs/Workshop), 2011.

[15] A. Joly and O. Buisson. A posteriori multi-probe locality sensitive hashing. In 16th ACM international conference on Multimedia, pages 209-218, 2008.

[16] A. Joly and O. Buisson. Random maximum margin hashing. In $C V P R$, pages 873-880, 2011.

[17] T. Kunii, C. Im, and H. Nishida. Recognizing plant species by leaf shapes: A case study of the acer family. In 14 th International Conference on Pattern Recognition (ICPR), pages Vol II: 1171-1173, 1998.

[18] H. Ling and D. Jacobs. Shape classification using the inner-distance. IEEE Transactions on Pattern Analysis and Machine Intelligence, 29(2):286 -299, Feb. 2007.

[19] Y. Mingqiang, K. Kidiyo, and R. Joseph. A survey of shape feature extraction techniques. Pattern Recognition Techniques, Technology and Applications, 2008. ISBN: 978-953-7619-24-4, InTech.

[20] F. Mokhtarian and S. Abbasi. Matching shapes with self-intersections: application to leaf classification. IEEE Transactions on Image Processing, 13(5):653 -661, May 2004.

[21] F. Mokhtarian, S. Abbasi, and J. Kittler. Robust and efficient shape indexing through curvature scale space. In British Machine Vision Conference (BMVC), 1996.

[22] G. Mori, S. J. Belongie, and J. Malik. Efficient shape matching using shape contexts. IEEE Trans. Pattern Anal. Mach. Intell., 27(11):1832-1837, 2005.

[23] Y. Nam, E. Hwang, and D. Kim. A similarity-based leaf image retrieval scheme: Joining shape and venation features. Computer Vision and Image Understanding, 110(2):245 - 259, 2008.

[24] J. C. Neto, G. E. Meyer, D. D. Jones, and A. K. Samal. Plant species identification using elliptic 
Fourier leaf shape analysis. Computers and Electronics in Agriculture, 50(2):121 - 134, 2006.

[25] J. Park, E. Hwang, and Y. Nam. Utilizing venation features for efficient leaf image retrieval. Journal of Systems and Software, 81(1):71 - 82, 2008.

[26] L. Paulevé, H. Jégou, and L. Amsaleg. Locality sensitive hashing: A comparison of hash function types and querying mechanisms. Pattern Recognition Letters, 31(11):1348-1358, 2010.

[27] M. Rusiñol and J. Lladós. Efficient logo retrieval through hashing shape context descriptors. In 9th IAPR International Workshop on Document Analysis Systems (DAS), pages 215-222, New York, NY, USA, 2010. ACM.

[28] E. Shechtman and M. Irani. Matching local self-similarities across images and videos. In IEEE Conference on Computer Vision and Pattern Recognition (CVPR), pages $1-8$, june 2007.

[29] Z. Wang, Z. Chi, and D. Feng. Shape based leaf image retrieval. IEE Proceedings on Vision, Image and Signal Processing, 150(1):34 - 43, Feb. 2003.

[30] J. Xie, P.-A. Heng, and M. Shah. Shape matching and modeling using skeletal context. Pattern Recogn., 41:1756-1767, May 2008.

[31] I. Yahiaoui, N. Hervé, and N. Boujemaa. Shape-based image retrieval in botanical collections. In 7th Pacific Rim Conference on Multimedia (PCM), volume 4261, pages 357-364, 2006.

[32] B. A. Yanikoglu, E. Aptoula, and C. Tirkaz. Sabanci-Okan system at ImageClef 2011: Plant identification task. In CLEF (Notebook Papers/Labs/Workshop), 2011. 